\title{
Detecting both melanoma depth and volume in vivo with a handheld photoacoustic probe
}

Yong Zhou, Guo Li, Liren Zhu, Chiye Li, Lynn A. Cornelius, et al.

Yong Zhou, Guo Li, Liren Zhu, Chiye Li, Lynn A. Cornelius, Lihong V. Wang, "Detecting both melanoma depth and volume in vivo with a handheld photoacoustic probe," Proc. SPIE 9708, Photons Plus Ultrasound: Imaging and Sensing 2016, 970806 (15 March 2016); doi: 10.1117/12.2213245

SPIE. Event: SPIE BiOS, 2016, San Francisco, California, United States 


\title{
Detecting Both Melanoma Depth and Volume in Vivo with a Handheld Photoacoustic Probe
}

\author{
Yong Zhou, ${ }^{a}$ Guo Li, ${ }^{a}$ Liren Zhu, ${ }^{a}$ Chiye Li, ${ }^{a}$ Lynn A. Cornelius, ${ }^{b}$ and Lihong V. Wang ${ }^{a^{*}}$ \\ ${ }^{a}$ Optical Imaging Laboratory, Department of Biomedical Engineering, Washington University in \\ St. Louis, 1 Brookings Drive, Campus Box 1097, St. Louis, Missouri 63130 \\ ${ }^{b}$ Division of Dermatology, Washington University School of Medicine, 660 S. Euclid, Campus \\ Box 8123, St. Louis, Missouri 63110 \\ *Correspondence: lhwang@wustl.edu
}

\begin{abstract}
We applied a linear-array-based photoacoustic probe to detect the tumor depth and volume of melanin-containing melanoma in nude mice in vivo. We demonstrated the ability of this linear-array-based system to measure both the depth and volume of melanoma through phantom, ex vivo, and in vivo experiments. The volume detection ability also enables us to accurately calculate the rate of growth of the tumor, which is important in quantifying tumor activity. Our results show that this system can be used for clinical melanoma diagnosis and treatment at the bedside. Keywords: Handheld, photoacoustic microscope, melanoma, depth, volume, rate of growth
\end{abstract}

\section{INTRODUCTION}

Melanoma is the most serious form of skin cancer. The incidence of melanoma has increased markedly in fairskinned populations living in industrialized countries since the 1970's. ${ }^{1}$ In the United States, it is now the 4th most common cancer in men and 5th most common cancer in women. In addition, it is estimated that the lifetime risk of developing melanoma for persons born in the year 2014 will be 1 in $50{ }^{2}$ Early diagnosis is the key, because melanoma is essentially curable if diagnosed when it is localized and non-invasive, but is fatal when metastatic. Currently, the standard of care is skin examination and recognition of gross lesion characteristics, followed by biopsy and histological examination of any suspicious lesions.

In the clinical setting, pigmented lesions that are concerning for melanoma may oftentimes be partially biopsied (either by tangential or punch biopsy), particularly in the cosmetically sensitive areas of the faces, hands, or feet. In these instances, if a diagnosis of melanoma is made on partial biopsy, a provisional Breslow's depth may be reported, as only part of the tumor is available for microscopic evaluation. A recent study shows that approximately $14 \%$ of all melanoma specimens were partially biopsied, leading not only to significant misdiagnosis, but also to microstaging inaccuracy. ${ }^{3}$ The same group had earlier demonstrated that in the hands of experienced dermatologists, accuracy of tumor depth by partial biopsy was achieved in only $88 \%$ of samples, which is unacceptable for appropriate patient management. ${ }^{4}$ In addition, in the current health care environment, many patients with dermatologic disease will be seen by physicians instead of by dermatologists, and the use of physician assistants is increasing: all of these contribute to a current trend, recognized several years ago, of inadequate technique and biopsy size estimations. ${ }^{5}$ In sum, the surgeon is often faced with a treatment dilemma in the case of a partially biopsied melanoma - whether to plan a "definitive" surgery based upon a provisional measurement in the anticipation that the Breslow's depth measurement represents the thickest portion of the tumor, or perform an excisional biopsy of the entire remaining lesion to get an accurate measurement prior to definitive surgery. ${ }^{6,7}$ Thus, in vivo imaging of melanomas to determine tumor depth could significantly aid melanoma patient management and alleviate the need for a second, excisional biopsy.

Combining both optical contrast and acoustic penetration, photoacoustic (PA) tomography (PAT) has been successfully applied in cutaneous vasculature imaging in humans in vivo. ${ }^{8-11}$ In PAT, the target is first illuminated with a short laser pulse. Then the laser light is absorbed by the target, leading to a temperature rise and a subsequent

Photons Plus Ultrasound: Imaging and Sensing 2016, edited by Alexander A. Oraevsky, Lihong V. Wang Proc. of SPIE Vol. 9708, 970806 - (C) 2016 SPIE · CCC code: 1605-7422/16/\$18 - doi: 10.1117/12.2213245 
initial pressure rise. ${ }^{12-17}$ The pressure rise propagates as a photoacoustic wave and is finally detected by either a focused ultrasonic transducer or a transducer array. Because melanin has strong optical absorption over a broad spectrum, it can be detected by PAT with high contrast. Meanwhile, high resolution can be maintained in PAT because the photoacoustic wave has low scattering in tissue. ${ }^{18}$

We recently reported that, in a murine xenograft melanoma model, tumor thickness can be accurately determined in vivo using a handheld photoacoustic probe. ${ }^{6}$ In that study, a single ultrasonic transducer and a $10 \mathrm{~Hz}$ laser were employed, limiting the imaging speed to around one frame per ten seconds. In addition, due to the limited view angle of the focused transducer, only the horizontal boundaries of the tumor could be detected. To address these limitations, we next employed an ultrasonic transducer-array-based photoacoustic probe to detect melanoma depth, as described herein. The imaging speed can be increased to five frames per second by using a $20 \mathrm{~Hz}$ laser. Using this probe and employing the large acceptance angle of the transducer array in the imaging plane, nearly the entire perimeter of the melanoma could be assessed. Furthermore, by simply adding a one-dimensional scanning stage, three-dimensional (3D) measurements of the melanoma could be obtained while the small footprint and convenience of the handheld device are maintained. With its ability to detect the margins in three axes, the array-based probe can be further used to determine the volume of a melanoma. It has been proposed that, similar to other solid tumors, melanoma volume may prove to be an excellent prognostic indicator - probably superior to Breslow's depth. ${ }^{19}$ In this manuscript, we first theoretically demonstrate that the linear-array-based probe can be used to determine and measure nearly the entire melanoma boundary, while the single ultrasonic transducer-based probe can detect only the horizontal boundary. Then we experimentally prove the ability of the linear-array-based probe to measure melanomas in phantoms, ex vivo, and in vivo.

\section{IMAGING SYSTEM}

A linear-array transducer (LZ250, Visualsonics Inc.), with a central frequency of $21 \mathrm{MHz}$ and a $55 \%$ bandwidth, detected the photoacoustic signals. ${ }^{20}$ The transducer array contained 256 elements, and had a size of $23 \mathrm{~mm} \times 3 \mathrm{~mm}$. A tunable optical parametric oscillator laser with a $20 \mathrm{~Hz}$ pulse repetition rate was the light source and was coupled into an optical fiber bundle. In our experiments, for melanoma imaging, the wavelength was set to $680 \mathrm{~nm}$, where melanin absorbs much more strongly than blood. The optical fiber bundle and an ultrasound cable were incorporated into the same package with the linear transducer array. The beam incident angle was $30^{\circ}$ with respect to the imaging plane. The optical fluence on the skin surface was estimated to be $10 \mathrm{~mJ} / \mathrm{cm}^{2}$, which was less than the safety limit set by the American National Standard Institute (ANSI) $\left(20 \mathrm{~mJ} / \mathrm{cm}^{2}\right)$ at this wavelength. Each laser pulse generated a two-dimensional image. To get a 3D PA image of the melanoma, the transducer array was scanned linearly along its elevational direction (x axis) by a motorized stage. An imaging station (Vevo LAZR, Visualsonics Inc.) displayed the photoacoustic images at five frames/second. The raw data was then exported to a computer for image reconstruction and analysis.

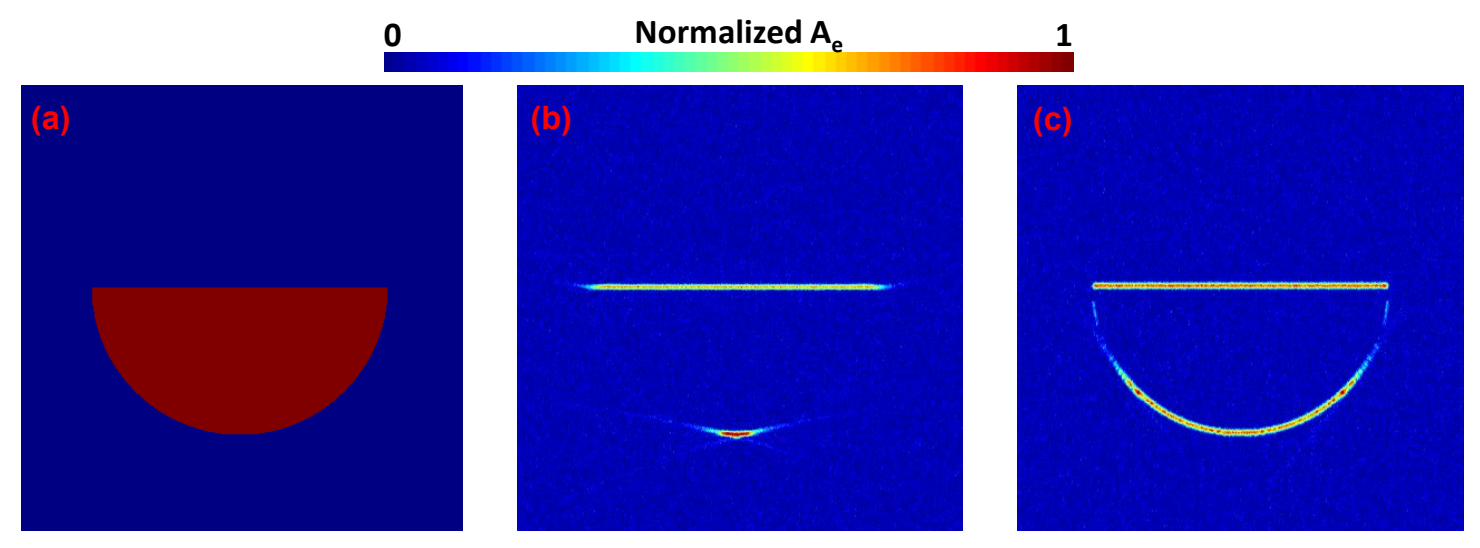

Fig. 1. (a) Preset energy deposition distribution $\left(A_{e}\right)$. (b) $A_{e}$ Recovered by a single normal transducer. (c) $A_{e}$ Recovered by linear array transducer. Image size: $6 \times 6 \mathrm{~mm}^{2}$. 


\section{SIMULATIONS}

Using the k-space pseudospectral method, ${ }^{21}$ we first simulated and compared the single focused ultrasonic transducer described previously ${ }^{6}$ with the array used in this paper. For the single focused ultrasonic transducer, the central frequency, bandwidth, focal length, and diameter were $25 \mathrm{MHz}, 100 \%, 12.7 \mathrm{~mm}$, and $6.4 \mathrm{~mm}$, respectively. The parameters of the array have been described above. Fig. 1(a) shows the melanoma-mimicking target, a half disk with a radius of $2 \mathrm{~mm}$. To form the image, we raster scanned the single focused ultrasonic transducer, but used a time reversal reconstruction algorithm for the array system. ${ }^{22}$ Fig. 1(b) shows that only the horizontal boundaries of the target are recovered by the single transducer based system. In contrast, almost the entire boundary of the target is recovered by the linear array (Fig. 1c). In addition, because the linear array scan does not need to be performed, the imaging speed can be significantly improved.
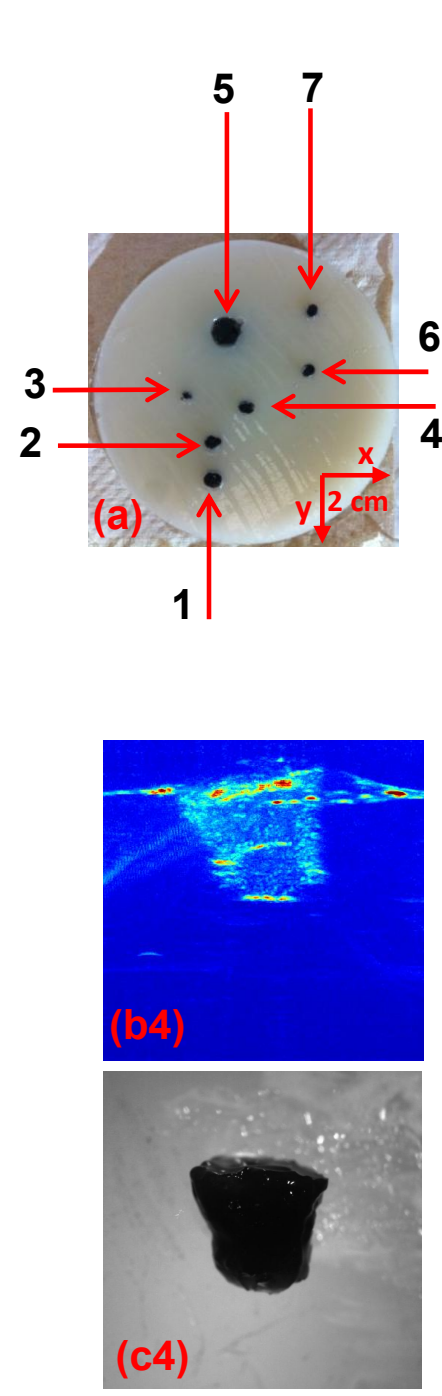

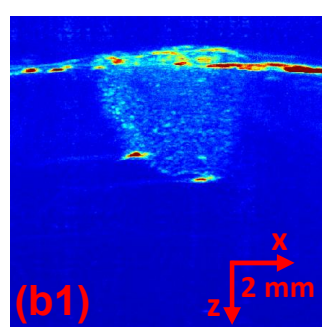

6
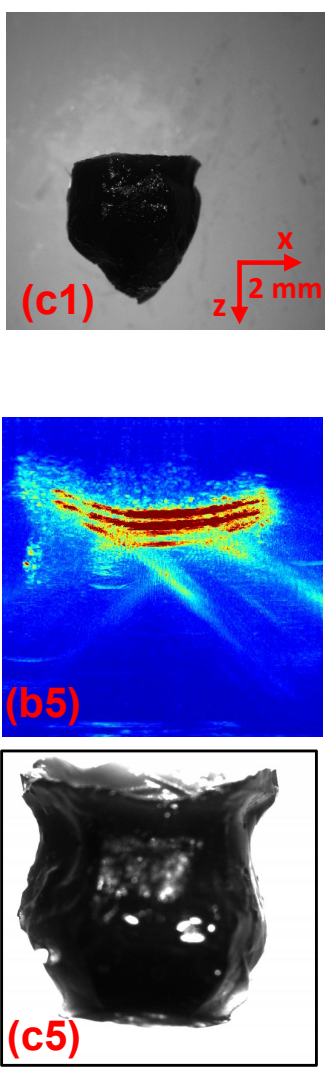
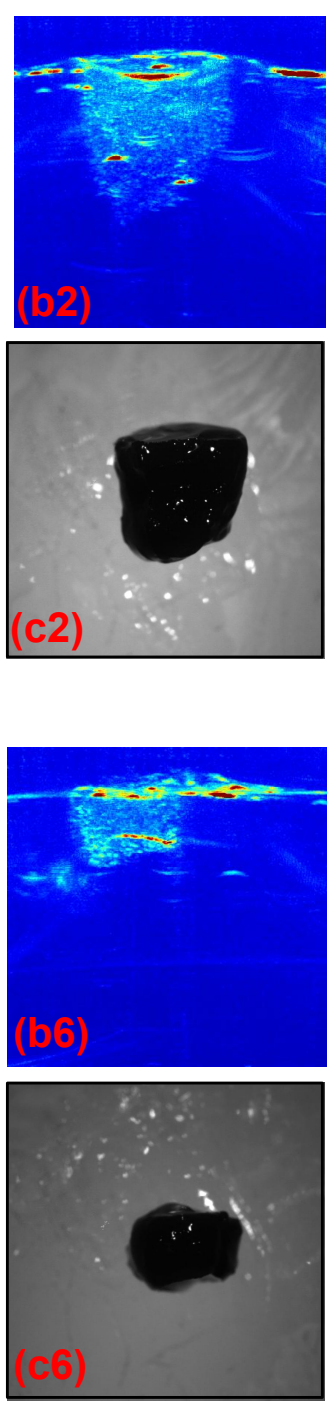
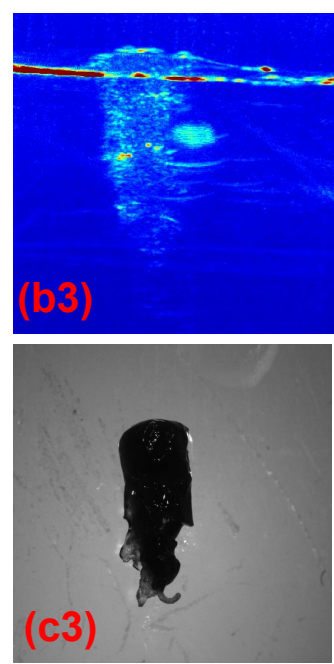

1
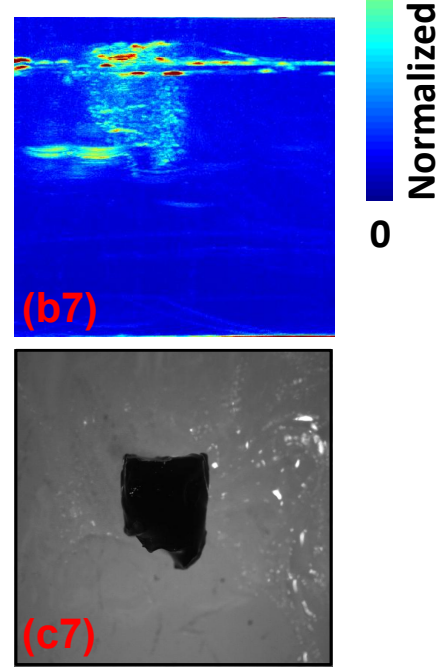

Fig. 2. Linear-array-based PA images of melanoma phantoms. (a) Photo of the melanoma phantoms. (b1-b7) and (c1-c7) correspond to the melanoma phantoms 1-7 in (a). Melanoma phantom images acquired by PA (b1-b7) and a standard optical microscope (c1-c7). 

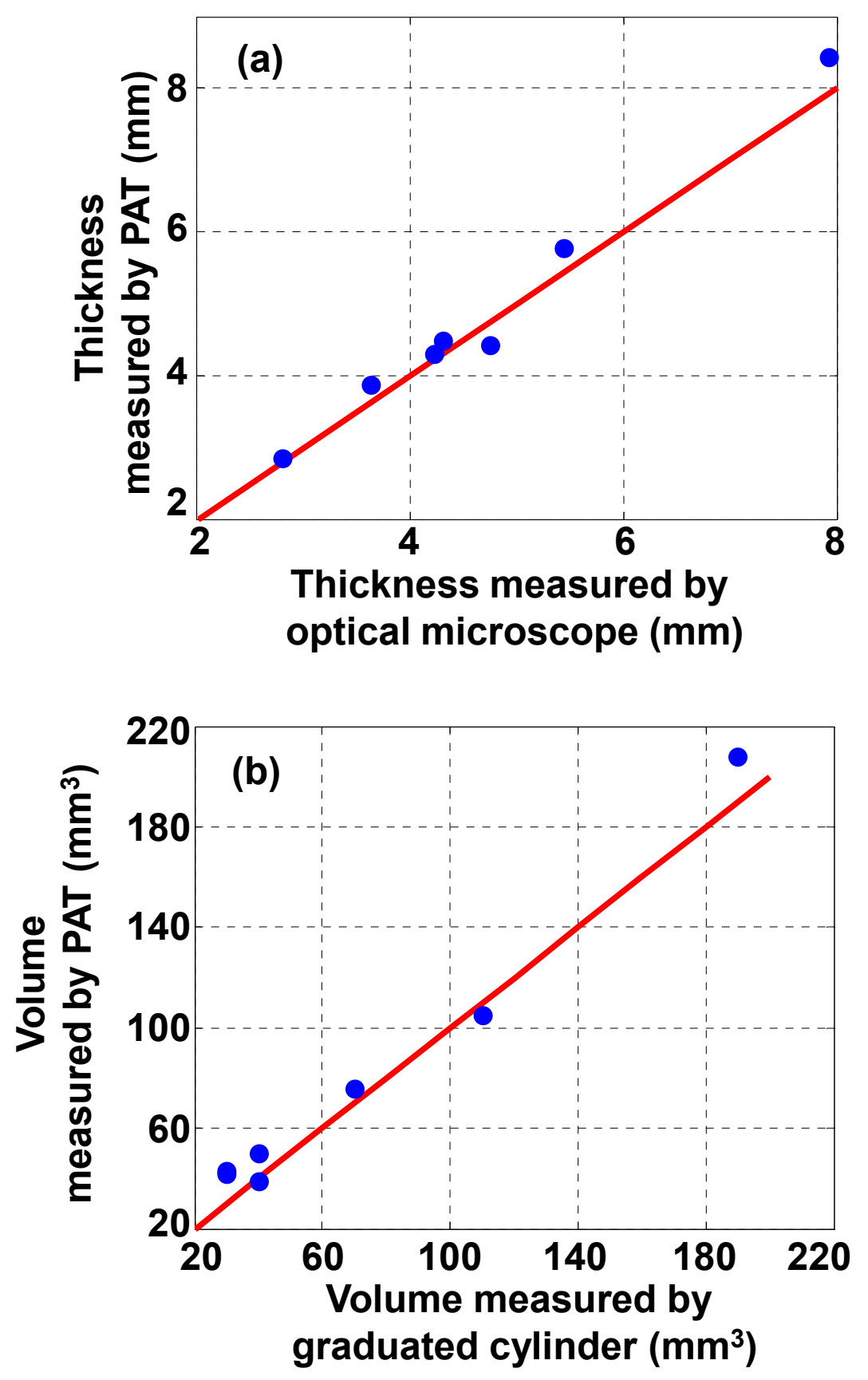

Fig. 3. Depth (a) and volume (b) quantification of melanoma phantoms. Blue dots, experimental measurements. Red lines, ideal fit if the PA measurements are identical to the standard measurements

\section{PHANTOM EXPERIMENTS}

Phantom experiments were then conducted to show the ability of the linear-array-based PA probe to measure melanoma thickness (depth) and enable calculating of the tumor volume (size). As shown in Fig. 2(a), melanoma phantoms were prepared with varying thicknesses and volumes. All the phantoms were made from a black ink and agar mixture, which had an absorption coefficient of $70 \mathrm{~cm}^{-1}$ at $680 \mathrm{~nm}$, close to the actual typical melanoma absorption coefficient. To mimic human tissue, the background was made of an agar and intralipid mixture with a 
scattering coefficient around $100 \mathrm{~cm}^{-1}$ and a scattering anisotropy of $\sim 0.9$. To facilitate optimal comparison of melanoma phantoms, we took the maximum-amplitude-projections of the 3D PA images along the elevational direction of the linear array, as shown in Fig. 2(b1-b7). Immediately following the array-based experiments, melanoma phantoms were carefully removed from the agar/intralipid mixture, and corresponding gross images were taken using a standard microscope (EZ4 Stereo microscope, Leica), as shown in Fig. 2(c1-c7). There is excellent correlation between the PA and gross images, demonstrating the ability of this system to accurately reproduce the phantom in situ. To further demonstrate the accuracy of the PA system and thus its potential for in vivo imaging, we then calculated both the depth and volume of these melanoma phantoms (Fig. 3). The thickness and volume of the melanoma phantoms measured by the standard microscope and a graduated cylinder, respectively, served as the gold standards. Both the depths and volumes measured by our system agree well with the standard values, indicating that our linear-array-based PA probe can detect both the depth and volume of deeply seated melanoma in situ with high accuracy.
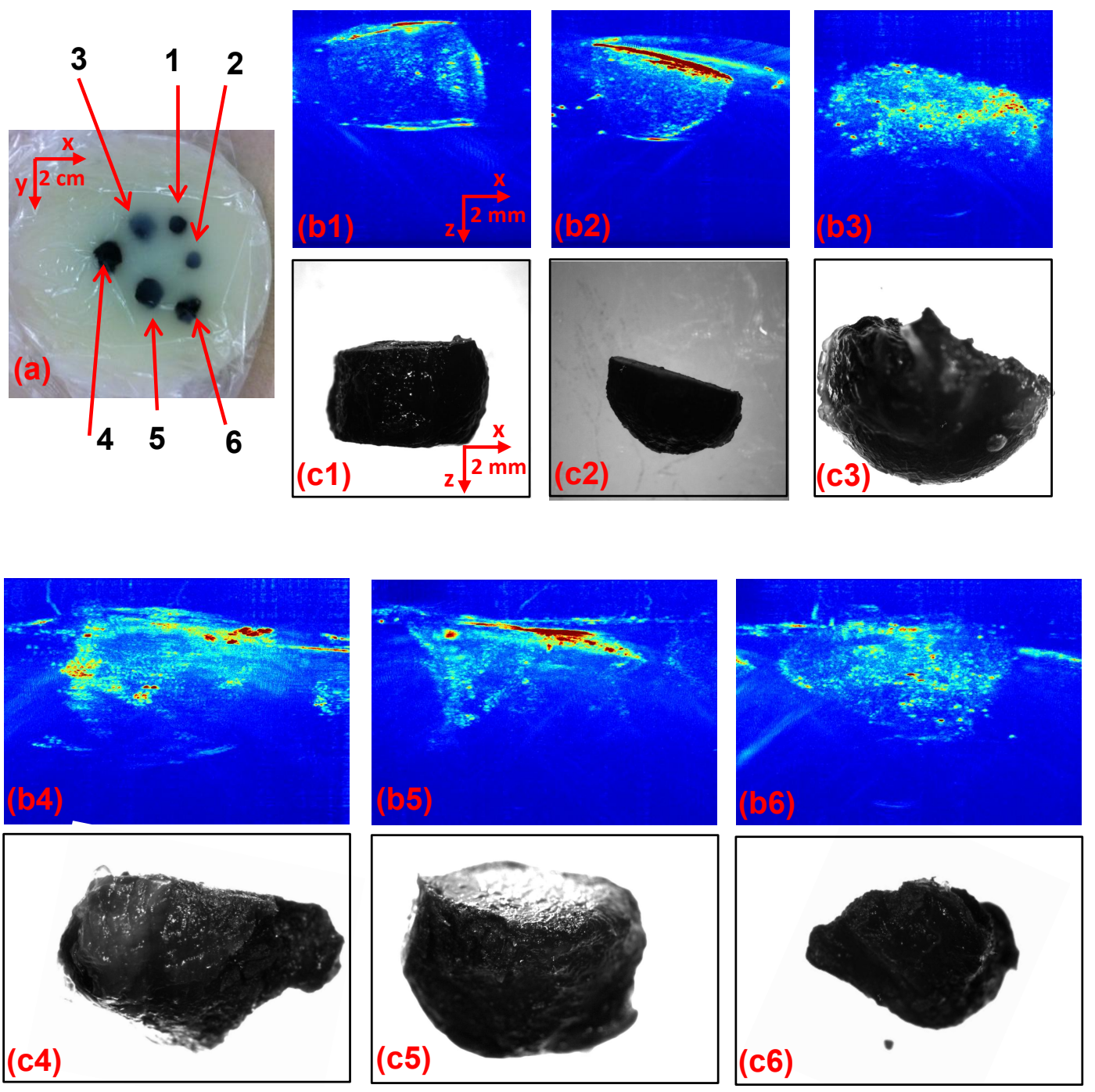

Fig. 4. Linear-array-based PA probe of ex vivo melanomas. (a) A photo of the ex vivo melanomas. Melanoma images acquired by PA (b1-g1) and a standard optical microscope (b2-g2). (b-g) correspond to the melanoma phantoms 1-6 in (a), respectively. 


\section{EX VIVO EXPERIMENTS}

We then performed experiments ex vivo to determine the accuracy of our system for thickness (depth) measurement and volume calculation. For these experiments, we used a nude murine xenograft model where B16 melanoma cells were subcutaneously injected into the dorsal surface (Hsd:Athymic Nude-Foxn1NU, Harlan Co.; average body weight, $20 \mathrm{~g}$ ). All experimental animal procedures were carried out in conformity with the laboratory animal protocol approved by the Animal Studies Committee of Washington University in St. Louis. Tumors were allowed to grow spontaneously for 15 days, and the mice were then sacrificed. Fresh tumors excised from nude mice were embedded in the agar and intralipid mixture, prepared as described earlier (Fig. 4a). Imaging and processing was then performed as in the phantom experiments. The ex vivo PA images of the melanomas are representative of the tumors, as shown in Figs. 4(b) and (c). Again, the thickness and volume values calculated from the images are in accordance with the preset values (shown in Fig. 5). Thus, we conclude that our system should be able to accurately image deep melanomas in vivo.
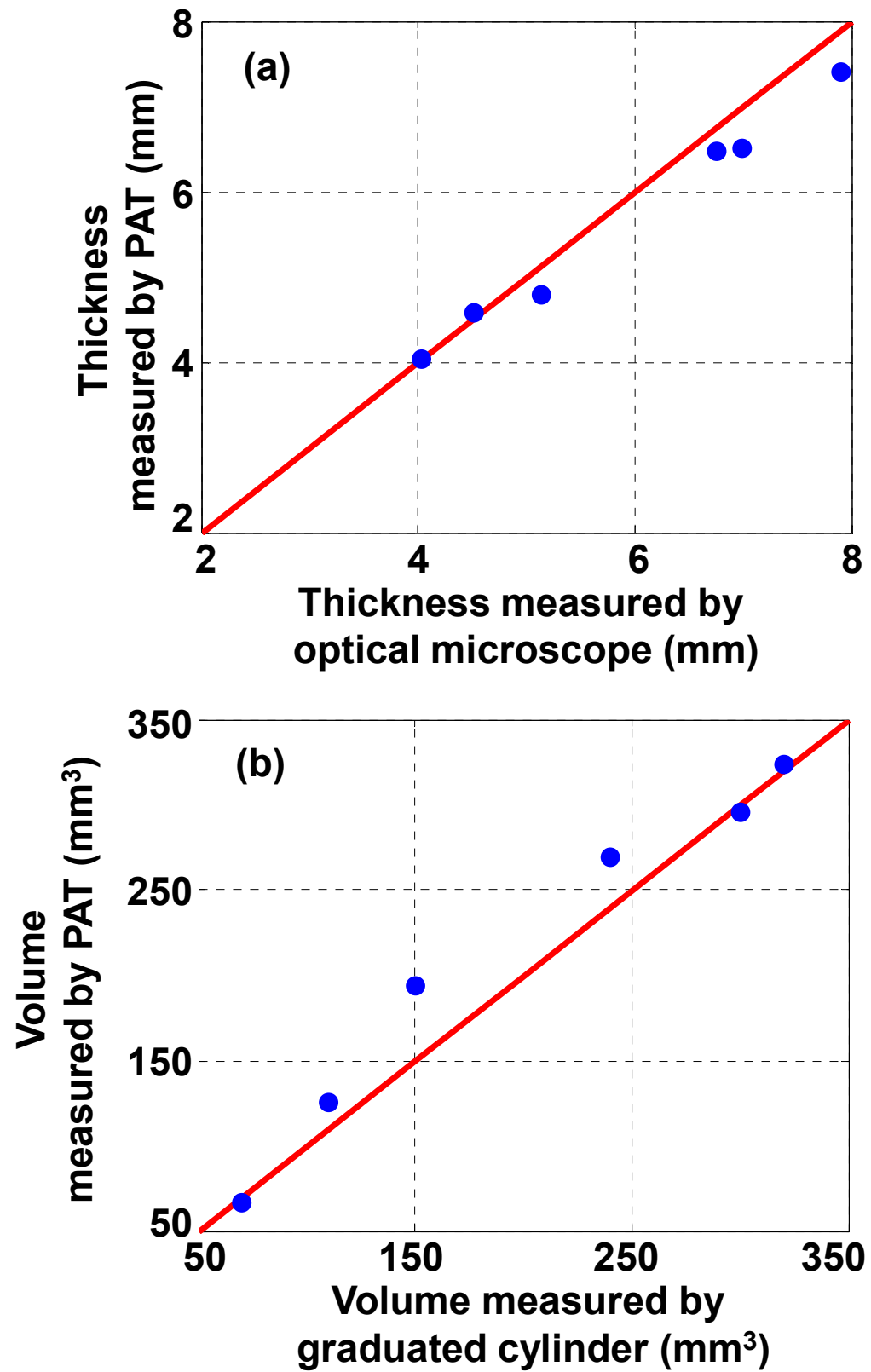

Fig. 5. Depth (a) and volume (b) quantification of melanoma phantoms. Blue dots, experimental measurements. Red lines, ideal fit if the PA measurements are identical to the standard measurements. 


\section{IN VIVO EXPERIMENTS}

Finally, we imaged mice with melanoma in vivo to show the detection ability of the linear-array-based PA probe. We again employed the nude murine xenograft model using B16 melanoma cells. For image acquisition, mice were anesthetized and kept motionless using a breathing anesthesia system (E-Z Anesthesia, Euthanex), while their body temperature was maintained by an electric heating pad. Each measurement was repeated at day 3 and day 6 following the injection of cells (Fig. 6a, b), and the rate of growth (ROG) was calculated. The detected melanoma can again be clearly distinguished from the surrounding soft tissue. The depth and volume of the melanoma at day 3 and day 6 were measured to be $1.32 \mathrm{~mm}$ and $22.4 \mathrm{~mm}^{3}$, and $2.77 \mathrm{~mm}$ and $71.9 \mathrm{~mm}^{3}$, respectively. The depth-based and volume-based ROG were calculated to be $0.48 \mathrm{~mm} /$ day and $16.5 \mathrm{~mm}^{3} /$ day, respectively.

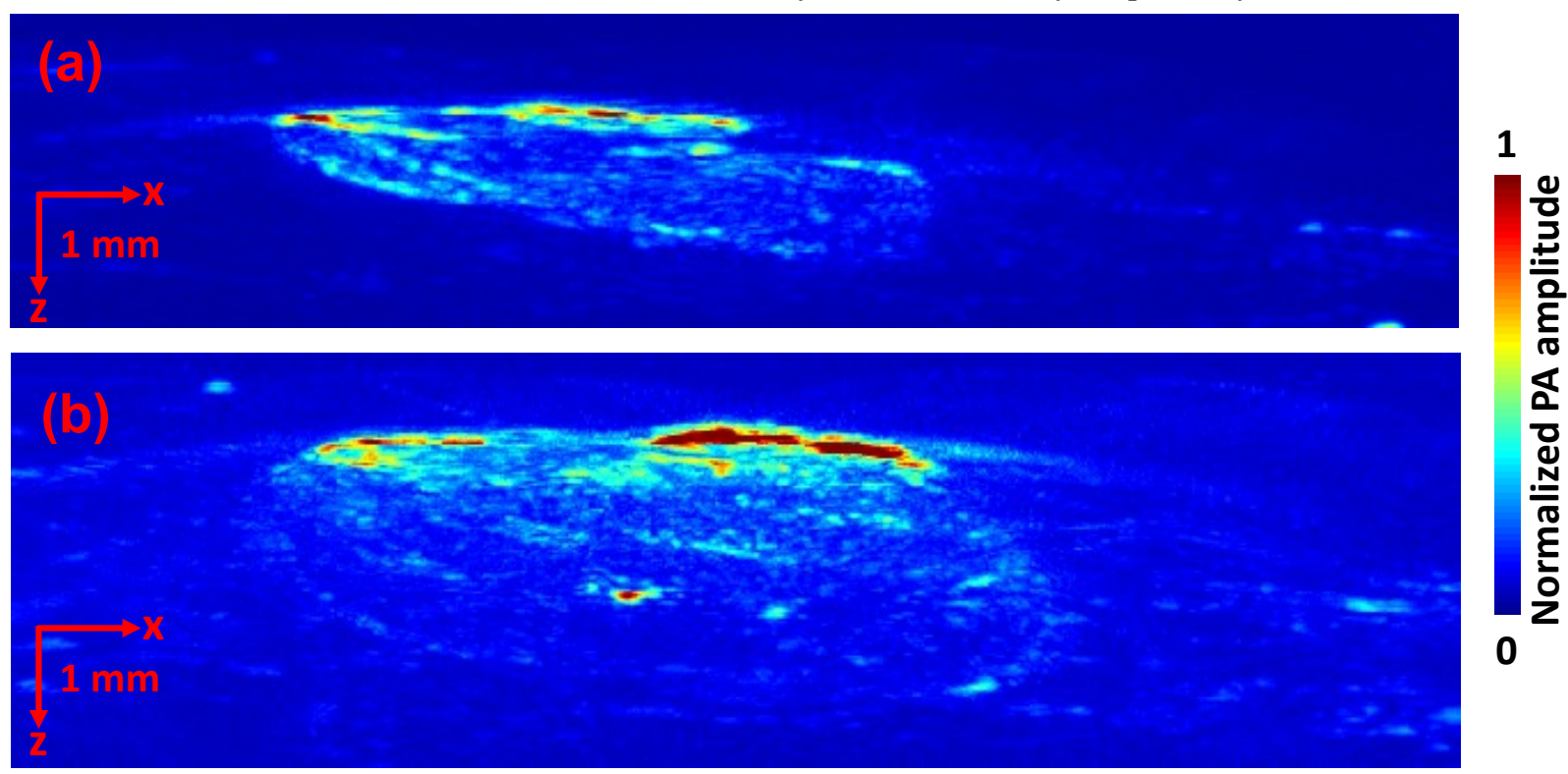

Fig. 6. Linear-array-based PA probe of melanoma in vivo. Melanoma image acquired at day 3 (a) and day 6 (b).

\section{DISCUSSION AND CONCLUSIONS}

In sum, surpassing our single ultrasonic transducer based system $^{6}$, the linear-array-based system can detect melanoma with an order of magnitude increase in the imaging speed. In addition, because of the large acceptance angle of the linear ultrasonic transducer array, nearly the entire melanoma can be detected in 3D, as opposed to only the horizontal boundary. Employing this technology also enables the calculation of melanoma volume. We hypothesize that, similar to other solid tumors, determination of melanoma volume may represent a more accurate diagnostic and prognostic parameter than the currently used measurement of tumor thickness (Breslow's depth). Furthermore, the ability to determine melanoma volume also provides a more straightforward and accurate way to determine and quantify change in a pigmented lesion that may be indicative of malignancy. We recognize that this increased accuracy comes at a price, considering the cost differential between the array system and the single ultrasonic transducer system. Nevertheless, we are now well poised to apply this system in the clinic to determine whether we can reproduce our findings in patients with pigmented lesions and melanoma. We propose that the increased accuracy afforded by the array will translate into improved management of the melanoma patient.

\section{ACKNOWLEDGEMENTS}

The authors would like to thank Prof. James Ballard for manuscript editing. This work was sponsored in part by National Institutes of Health grants DP1 EB016986 (NIH Director's Pioneer Award), R01 CA186567 (NIH Director's Transformative Research Award), R01 EB016963, S10 RR026922, and R01 CA159959. L.W. has a financial interest in Microphotoacoustics, Inc. and Endra, Inc., which, however, did not support this work. 


\section{REFERENCES}

[1] A. Jemal, S. S. Devesa, P. Hartge, and M. A. Tucker, "Recent trends in cutaneous melanoma incidence among whites in the United States," J. Natl. Cancer Inst. 93 (9), 678-683 (2001).

[2] American Cancer Society, http://www.cancer.org/.

[3] J. C. Ng, S. Swain, J. P. Dowling, R. Wolfe, P. Simpson, and J. W. Kelly, "The impact of partial biopsy on histopathologic diagnosis of cutaneous melanoma: experience of an Australian tertiary regerral service," Arch. Dermatol. 146 (3), 234-239 (2010).

[4] P. C. Ng, D. A. Barzilai, S. A. Ismail, R. L. Averitte, Jr., and A. C. Gilliam, "Eveluating invasive cutaneous melanoma: is the initial biopsy representative of the final depth?" J. Am. Acad. Dermatol. 48 (3), 420-424 (2003).

[5] K. Sellheyer, P. Nelson, and W. Bergfeld, "Inadequate biopsy technique and specimen size: an alarming tread that compromises patient care and an appeal to our clinical colleagues," Arch. Dermatol. 146 (10), 1180-1181 (2010).

[6] Y. Zhou, W. Xing, K. I. Maslov, L. A. Cornelius, and L. V. Wang, "Handheld photoacoustic microscopy to detect melanoma depth in vivo," Opt. Lett. 39 (16), 4731-4734 (2014).

[7] Y. Zhou, G. Li, L. Zhu, C. Li, L. A. Cornelius, and L. V. Wang, "Handheld photoacoustic probe to detect both melanoma depth and volume at high speed in vivo," J. Biophotonics 8(11-12), 961-967 (2015).

[8] Y. Zhou, X. Yi, W. Xing, S. Hu, K. I. Maslov, and L. V. Wang, "Microcirculatory changes identified by photoacoustic microscopy in patients with complex regional pain syndrome type I after stellate ganglion blocks," J. Biomed. Opt. 19 (8), 086017 (2014).

[9] H. F. Zhang, K. Maslov, G. Stoica, and L. V. Wang, "Functional photoacoustic microscopy for highresolution and noninvasive in vivo imaging," Nat. Biotechnol. 24 (7), 848 (2006).

[10] P. Hai, Y. Zhou, J. Liang, C. Li, and L. V. Wang, "Photoacoustic tomography of vascular compliance in humans," J. Biomed. Opt. 20(12), 126008 (2015).

[11] Y. Zhou, J. Liang, and L. V. Wang, "Cuffing-based photoacoustic flowmetry in humans in the optical diffusive regime," J. Biophotonics 1-5 (2015), DOI 10.1002.

[12] Y. Zhou, J. Liang, K. I. Maslov, and L. V. Wang, "Calibration-free in vivo transverse blood flowmetry based on cross correlation of slow time profiles from photoacoustic microscopy," Opt. Lett. 38 (19), 3882-3885 (2013).

[13] L. V. Wang and L. Gao, "Photoacoustic Microscopy and Computed Tomography: From Bench to Bedside," Annu. Rev. Biomed. Eng. 16, 155-85 (2014).

[14] Y. Zhou, C. Zhang, D.-K. Yao, and L. V. Wang, "Photoacoustic microscopy of bilirubin in tissue phantoms," J. Biomed. Opt. 17 (12), 126019 (2012).

[15] P. Hai, J. Yao, K. I. Maslov, Y. Zhou, and L. V. Wang, "Near-infrared Optical-resolution Photoacoustic Microscopy," Opt. Lett. 39 (17), 5192-5195 (2014).

[16] L. V. Wang and S. Hu, "Photoacoustic tomography: in vivo imaging from organelles to organs," Science 335 (6075), 1458-1462 (2012).

[17] Y. Zhou, J. Yao, K. I. Maslov and L. V. Wang, "Calibration-free absolute quantification of particle concentration by statistical analyses of photoacoustic signals in vivo," J. Biomed. Opt. 19 (3), 37001 (2014).

[18] Y. Zhou, J. Yao, and L. V. Wang, "Optical clearing-aided photoacoustic microscopy with enhanced resolution and imaging depth," Opt. lett. 38(14), 2592-2595 (2013).

[19] W. Liu, J. P. Dowling, W. K. Murray, G. A. McArthur, J. F. Thompson, R. Wolfe, and J. W. Kelly, "Rate of growth in melanomas," Arch. Dermatol. 142 (12), 1551-1558 (2006).

[20] A. Needles, A. Heinmiller, J. Sun, C. Theodoropoulos, D. Bates, D. Hirson, M. Yin, and F. S. Foster, "Development and initial application of a fully integrated photoacoustic micro-ultrasound system," IEEE Trans. Ultrason. Ferroelectr. Freq. Control 60(5), 888-97 (2013).

[21] B. E. Treeby, J. Jaros, A. P. Rendell, and B. T. Cox, "Modeling nonlinear ultrasound propagation in heterogeneous media with power law absorption using a k-space pseudospectral method," J. Acoust. Soc. Am. 131 (6), 4324-4336 (2012).

[22] B. E. Treeby and B. T. Cox, "k-Wave: MATLAB toolbox for the simulation and reconstruction of photoacoustic wave-fields," J. Biomed. Opt. 15 (2), 021314 (2010). 